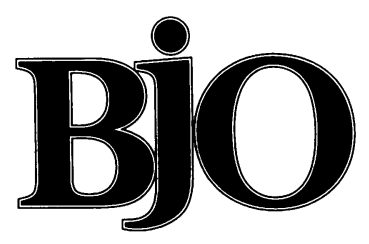

British Journal of Ophthalmology

Amblyopia continues to attract tremendous interest from basic scientists and clinicians, yet surprisingly large gaps in our understanding of this topic remain. One of these is highlighted by Abrahamsson and Sjöstrand who describe in this issue of the $B F O(p 860)$ a meticulous follow up of 20 children to the age of 10 who at 1 year of age had $\geq 3.0$ dioptres of anisometropia. Three distinct patterns of development emerged. Firstly, in some the anisometropia decreased (mean 3.0 DS) and no amblyopia resulted. A second group all developed amblyopia and the anisometropia increased (mean 1.4 DS), while in a third group the anisometropia decreased to a lesser extent than in the first group (mean 1.2 DS) and all developed squint and/or amblyopia. At the outset, all infants studied had received full refractive correction so one could quibble with the authors' use of the term 'natural history' in referring to the development they observed. Nevertheless, their work draws attention to how little we know about the development of abnormal refractive states and, specifically, anisometropia. The question now posed is which occurs first, amblyopia or anisometropia? Hitherto, it has been generally assumed that anisometropia precedes amblyopia; indeed, this is said to differentiate the amblyopia of microtropia from that occurring in larger angle strabismus. ${ }^{1}$ However, the direction of causality in this relation has been questioned. ${ }^{2}$ Six years ago Almeder and colleagues wrote '...despite the clear association of anisometropia with amblyopia and despite demonstrations from animal models that anisometropia can cause amblyopia, it has not been proven that the anisometropia seen in amblyopic school children or adults is the cause of that amblyopia." By 1992 an alternative opinion was forming '...we are beginning to suspect that anisometropia, at least in the population we studied, may be more often a result than a cause of amblyopia. ${ }^{4}$ To most clinicians this is counterintuitive yet this hypothesis in fact arose out of clinical practice. Lepard ${ }^{5}$ and, later, Nastri and colleagues ${ }^{6}$ observed that in unilateral amblyopia the refractive status of the fixing eye becomes more myopic while the amblyopic eye remains hypermetropic. Refractive development studies in normal and strabismic children have shown that the incidence of anisometropia in infants can be as high as $25 \%$ and in early childhood is between $2.8 \%$ and $8 \% .^{7}$ While the prevalence of anisometropia throughout childhood is relatively constant, the children who make up this group are not, because anisometropia 'goes' and 'comes' ${ }^{37-10}$ It goes during the process of emmetropisation as exemplified by some of the children in Abrahamsson and Sjöstrand's study and this transient anisometropia of infancy and early childhood is of no significance for vision. On the other hand, anisometropia also comes as it can increase or develop with strabismus (eso not exo deviations) and/or amblyopia ${ }^{810}$; indeed, it is uncommon in older children without amblyopia. ${ }^{3}$ These findings have been confirmed experimentally by Kiorpes and Wallman ${ }^{11}$ who observed hypermetropic anisometropia in monkeys with surgically produced strabismus and amblyopia. Here, amblyopia preceded anisometropia. It is not clear from Abrahamsson and Sjöstrand's current paper whether the anisometropia was present before the development of amblyopia, although their, as yet, unpublished data (Abrahamsson $\mathrm{M}$, personal communication) indicates that in the majority of cases the latter comes first. Should this be confirmed then to some extent the need for refractive screening for anisometropia to prevent, rather than to treat, amblyopia will have been undermined and urgent consideration will need to be given to identifying the aetiological factors which result in amblyopia. Finally, if anisometropia turns out to be merely a secondary phenomenon in the genesis of amblyopia, then what of the implications for treatment? Current clinical practice consists of spectacle correction followed by occlusion therapy. On the basis of work with primates, the possible adverse influence of full spectacle correction on emmetropisation has recently been raised by Hung et al, ${ }^{12}$ and since vigorously debated by Stone et $a l,{ }^{13}$ while in practice Atkinson et al ${ }^{14}$ find partial spectacle correction not to interfere significantly with emmetropisation. According to current clinical dogma once amblyopia exists optical correction is not expected to contribute to its reduction. Our own findings suggest, however, that the correction of anisometropia as a precursor to occlusion therapy may in fact exert a therapeutic influence far greater than previously imagined. Spectacle induced acuity improvement was seen to continue for several months following correction, which exceeded the benefits of a minimal occlusion regimen ${ }^{15}$-a seemingly paradoxical finding were anisometropia but a consequence of amblyopia. Clearly, there is so much more to know about amblyopia, and the article by Abrahamsson and Sjöstrand has identified an issue to which few clinicians have given much thought: in children with amblyopia and anisometropia, which is the chicken and which is the egg? 
1 Helveston EM, von Noorden GK. Microtropia: a newly defined entity. Arch Ophthalmol 1967;78:272-81.

2 Ingram RM. Amblyopia. BMf 1989;298:204.

3 Almeder LM, Peck LB, Howland HC. Prevalence of anisometropia in volunteer laboratory and school screening populations. Invest Ophthalmol Vis Sci 1990;31:2448-55.

4 Howland HC. Early refractive development. In: Simons K, ed. Early visual development, normal and abnormal. New York: Oxford University Press, 1993:5-13.

5 Lepard CW. Comparative changes in the error of refraction between fixing and amblyopic eyes during growth and development. Am $\mathcal{f}$ Ophthalmol and amblyopic eyes

6 Nastri G, Caccia Perugini C, Savastano S, Polzella A, Sbordone G. The evolution of refraction in the fixing and the amblyopic eye. Doc Ophthalmol lution of refraction

7 Saunders KJ. Early refractive development in humans. Surv Ophthalmol 1995;40:207-16.

8 Ingram RM, Barr A. Changes in refraction between the ages of 1 and $31 / 2$ years. Br ₹ Ophthalmol 1979;63:339-42.
9 Abrahamsson M, Fabian G, Sjöstrand J. Changes in astigmatism between the ages of 1 and 4 years: a longitudinal study. $B r f$ Ophthalmol the ages of 1 .

10 Abrahamsson $M$, Fabian G, Sjöstrand J. Refraction changes in children developing convergent or divergent strabismus. $\mathrm{Br} \mathcal{F}$ Ophthalmol 1992;76: 723-7.

11 Kiorpes L, Wallman J. Does experimentally-induced amblyopia cause hyperopia in monkeys? Vision Res 1995;35:1289-97.

12 Hung L-F, Crawford MLJ, Smith EL. Spectacle lenses alter eye growth and the refractive status of young monkeys. Nature Med 1995;1:761-5.

13 Stone RA, Laties AM, Fernandes A, Tigges M. Spectacles and young eyes. Nature Med 1996;2:610-11.

14 Atkinson J, Braddick O, Bobier B, Anker S, Ehrlich D, King J, et al. Two infant vision screening programmes: prediction and prevention of infant vision screening programmes: prediction and prevention of strabismus and amb;10:189-98

15 Moseley MJ, Irwin M, Jones HS, Fielder AR, Auld R. Effect of spectacle wear and minimal occlusion therapy on the vision of amblyopic chlidren. Invest Ophthalmol Vis Sci 1996;37:S941.

\section{Blood pressure and glaucoma}

The paper by Meyer et al in this issue of the $B F O$ (p 864) adds another intriguing piece to the puzzle that represents the relation between blood pressure and glaucoma. Unfortunately, it often seems that we have an increasing number of pieces but less overall picture.

As long ago as 1857, von Graefe reported a patient with glaucomatous optic neuropathy but with a normal intraocular pressure (IOP), implying-even then-that glaucoma was about more than simply raised intraocular pressure. ${ }^{1}$ Wolff, ${ }^{2}$ in 1947 , speculated that glaucoma was the result of optic nerve ischaemia while Duke-Elder ${ }^{3}$ considered arteriosclerosis to be an important factor in open angle glaucoma.

Systemic hypertension has been implicated for many years as a risk factor for developing glaucoma ${ }^{45}$ but results have been plagued by the use of single blood pressure measurements (the 'white coat effect') and the bias of hospital based studies. Major advances in our understanding of the relation have only come about with the advent of large scale population based surveys and 24 hour ambulatory blood pressure monitoring.

We have known for some years that systemic blood pressure and IOP were significantly correlated and this has been confirmed by the Baltimore Eye Study. ${ }^{6}$ However, the association between blood pressure and glaucoma is not so clear cut. The Baltimore Eye Study did show a small positive association of primary open angle glaucoma with higher systolic and diastolic blood pressure, the relation was non-linear and the systolic threshold was at $130 \mathrm{~mm}$ $\mathrm{Hg}$ - above which the risk increased. They also suggested, by using age as a surrogate for duration of systemic hypertension, a protective effect of hypertension in those under 60 and an adverse effect among those over 70 with respect to primary open angle glaucoma. The Rotterdam study ${ }^{7}$ found an association between primary open angle glaucoma and systolic blood pressure but not normal tension glaucoma (perhaps explained by the blood pressure-IOP association), while the Barbados Eye Study ${ }^{8}$ concluded that high blood pressure alone was not a risk factor for primary open angle glaucoma but did indicate, like the Framingham Eye Study, ${ }^{9}$ that people with field defects had significantly decreased blood pressure/IOP ratios.

Simply comparing blood pressure in individuals with and without glaucoma is never going to give us a definitive answer. This led the Baltimore Eye Study investigators to calculate vascular perfusion pressure in their subjects-this being the blood pressure (systolic, diastolic, or mean) minus the IOP-and showed that the lower the diastolic perfusion pressure the higher the prevalence of primary open angle glaucoma. ${ }^{6}$ In fact, subjects with diastolic perfusion pressures below $30 \mathrm{~mm} \mathrm{Hg}$ had an age adjusted risk of POAG six times higher than those with pressures of 50 $\mathrm{mm} \mathrm{Hg}$ or greater.

However, the blood pressure measurements in the Baltimore Eye Study consisted of only two readings 5 minutes apart. It has been shown consistently that 24 hour ambulatory blood pressure monitoring gives lower readings than routine clinic measurements and correlates very well with direct intra-arterial recordings. ${ }^{10}$ There have now been a number of studies of 24 hour blood pressure monitoring in glaucomatous patients and the results are important.

Hayreh et al $^{10}$ compared 24 hour blood pressures in patients with normal tension glaucoma, primary open angle glaucoma, and anterior ischaemic optic neuropathy and found a larger drop in mean systolic and diastolic blood pressure at night in the group with normal tension. This concept of 'nocturnal dips' in blood pressure has only come to light using 24 hour blood pressure readings and is now well recognised by cardiovascular physicians. Studies have shown that if hypertensive patients have their diastolic blood pressure reduced below a critical level then their rate of adverse cardiac events increases. ${ }^{11}$ Hayreh et al ${ }^{10}$ extrapolated this to suggest that a similar process could occur in the optic nerve head and ocular ischaemic disorders.

Graham et al ${ }^{12}$ showed that all nocturnal blood pressure variables were lower in (normal tension glaucoma or primary open angle glaucoma) patients with progressive field loss than in those with stable fields. Bechetoille and Bresson-Dumont ${ }^{13}$ found a lower diastolic and systolic blood pressure in patients with normal tension glaucoma compared with those with primary open angle glaucoma and a greater percentage of diurnal low readings in the former group. Kaiser et $a l^{14}$ also indicated that arterial hypotension was a risk factor for glaucomatous damage. The paper by Meyer et al also shows that patients with normal tension glaucoma have larger nocturnal blood pressure drops than normal controls.

Two potential confounders of any of these studies are the use of systemic and/or ocular antihypertensives by the subjects being studied. Hayreh et al ${ }^{10}$ showed a significant association between visual field loss and nocturnal hypotension in patients taking systemic antihypertensive medications. Graham et al's paper ${ }^{12}$ suggested that the nocturnal dip was exacerbated by systemic antihypertensives, but did not show an effect caused by topical $\beta$ blockers.

The association of nocturnal blood pressure dips and 